\title{
miR-141 regulation of EIF4E expression affects docetaxel chemoresistance of non-small cell lung cancer
}

\author{
DONG WANG $^{1}$, JUNJIE MA ${ }^{2}$, XIANGYU JI $^{3}$, FENG XU $^{1}$ and YUCHENG WEI ${ }^{1}$ \\ ${ }^{1}$ Department of Thoracic Surgery, The Affiliated Hospital of Qingdao University, Qingdao, Shandong 266003; \\ ${ }^{2}$ Department of Thoracic Surgery, The Second People's Hospital of Liaocheng, Liaocheng, Shandong 252600; \\ ${ }^{3}$ Department of Anesthesia, The Affiliated Hospital of Qingdao University, Qingdao, Shandong 266003, P.R. China
}

Received April 11, 2016; Accepted August 16, 2016

DOI: $10.3892 /$ or.2016.5214

\begin{abstract}
The present study investigated the role of miR-141 regulation of eukaryotic initiation factor-4E (EIF4E) expression in docetaxel chemoresistance of human non-small cell lung cancer (NSCLC). The expression of miR-141 in docetaxel chemoresistant patients with NSCLCs was markedly higher than those of non-docetaxel chemoresistant patients with NSCLCs. The expression of EIF4E in docetaxel chemoresistant patients with NSCLCs was markedly lower than those of non-docetaxel chemoresistant patients with NSCLCs. Downregulation of miR-141 suppressed cell proliferation, induced cell death and increased caspase-3 activity in H1299 or H2009/docetaxel cells. Downregulation of miR-141 also increased the protein expression of EIF4E, VEGF, c-Myc and Bax in H1299 or H2009/docetaxel cells. Conversely, upregulation of miR-141 promoted cell proliferation, inhibited cell death and caspase-3 activity in H1299 or H2009/ docetaxel cell. Upregulation of miR-141 suppressed EIF4E, VEGF, c-Myc protein expression and inhibited Bax in H1299 or H2009/docetaxel cells. Thus, the present study is the first to show the induction of miR-141/EIF4E expression in an acquired model of docetaxel chemoresistant patients with NSCLCs. This serves as a mechanism of acquired docetaxel chemoresistant patients with NSCLCs, possibly through direct interactions with VEGF, c-Myc, and Bax, therefore presenting a potential therapeutic target for the treatment of docetaxel chemoresistant patients with NSCLCs.
\end{abstract}

\section{Introduction}

Lung cancer is one of the most common malignant tumors. According to the statistics data (GLOBOCAN) issued by the World Health Organization, lung cancer is the most common

Correspondence to: Dr Yucheng Wei, Department of Thoracic Surgery, The Affiliated Hospital of Qingdao University, 16 Jiangsu Road, Qingdao, Shandong 266003, P.R. China

E-mail: weiyuchengchest@163.com

Key words: miR-141, eukaryotic initiation factor-4E, non-small cell lung cancer cancer with the highest fatality rate for male in 2008; while for female, it is the fourth common one with the fatality rate ranked the second (1). In 2008, lung cancer accounted for $13 \%$ (1.6 million) of new onset cancer cases, and $18 \%$ of deaths (1.4 million) (2). Lung cancer is the malignant tumor with the highest morbidity in China as well. Its morbidity in 2003-2007 was $48.90 / 100,000$, accounting for $18.39 \%$ of all new onset cancer cases; lung cancer was ranked in the top position among regions with cancer registry in China in 2003-2007 (3). The mortality rate was $43.48 / 100,000$, accounting for $25.30 \%$ of all new onset cancer cases (4).

Similar to other chemotherapeutics, drug resistance to Taxanes has always been a great obstacle for further enhancing therapeutic effect of chemotherapy, prolonging patient lifetime and improving QOL. A variety of factors may lead to drug resistance to Taxanes, including gene mutation of $\alpha$-tubulin and p-tubulin, diversified constitutions of p-tubulin hypotype, overexpression of $\mathrm{P}$-gp and increasing of microtubule activity related to the changed microtubule-associated protein. In addition, among a variety of molecular pathways associated with cell cycle and cell apoptosis, some changes on protein expression may be associated with chemotherapy resistance.

Eukaryotic initiation factor-4E (EIF4E) is a protooncogene detected recently. It plays significant role at the initial period of protein synthesis (5). EIF4E overexpression can change expression quantity of malignant tumor related genes. It is the determinant of tumor malignant phenotype (6). It may make cells break through the limitation of normal growth thus leading to carcinogenesis by adjusting expression translation amount of specific malignant related molecules (such as VEGF, cylinD1 and C-myc) (7). Generally, a small amount of expression of eIF4E can maintain mRNA translation required by normal life activities of cells. In case of overexpression, it may lead to increasing of mRNA expression translation of a variety of protein coding carcinoma and tumor promoters thus to promote genesis and development of tumor. Research has shown that overexpression of eIF4E exists in many malignant tumors (such as: bladder cancer, cervical cancer, leukemia, head and neck malignant tumor, laryngeal cancer, breast cancer, esophagus cancer, colon cancer, prostatic cancer and bile duct carcinoma) $(7,8)$.

Research has verified that resistance mechanism involves a variety of factors, including drug accumulation reduction, drug 
detoxification increase, changes of drug-resistance-related genes, inhibition of tumor cell apoptosis and enhancement of DNA rehabilitation capacity, and involvement of multiple signal pathways and key cytokines (9). In the last two years, it has been detected that some miRNAs are related to sensitivity and drug resistance of chemotherapy. In case of expression upregulation or downregulation, it will directly lead to expression level abnormity of target gene protein in related pathways of drug resistance (10). Eventually, drug sensitivity of tumor cells is changed through signal pathway of the cell. Such target genes include those related to cell apoptosis, drug transporter, drug target, cell repairing and cell cycle regulation (11). At present, it has been detected that some miRNAs is associated with sensitivity of chemical drug treatment of tumors (12). The present study investigated the role of miR-141 regulation of EIF4E expression in docetaxel chemoresistance of NSCLCs.

\section{Materials and methods}

Patients. This study was conducted in accordance with the ethical principles of The Affiliated Hospital of Qingdao University. NSCLC patients had a histological diagnosis of NSCLC from The Affiliated Hospital of Qingdao University. All patients provided written informed consent before undergoing any study procedure. In total, docetaxel chemoresistant patients with NSCLCs was 16 and non-docetaxel chemoresistant patients with NSCLCs was 8 .

Real-time quantitative reverse transcription-PCR. Total RNA of tumor samples or cancer cells was isolated using the TRIzol reagent (Invitrogen). Reverse transcription (RT)PCR was accomplished by the PrimeScript RT Reagent kit (Takara Biotechnology Co., Ltd., Dalian, China) using 1-2 $\mu \mathrm{g}$ total RNA. Real-time quantitative reverse transcription-PCR was performed using SYBR Premix Ex Taq ${ }^{\mathrm{TM}}$ II (Takara Biotechnology Co., Ltd.). Expression mRNA levels of miR-141 and EIF4E were defined as positive controls for the $2^{-\Delta \Delta \mathrm{Ct}}$ calculation. The designed PCR primers of miR-141 and EIF4E are shown in Table I.

Cell culture. Human NSCLC H1299 and H2009 cells were from the Cell Bank of Chinese Academy of Medical Science. Cells were cultured and maintained in RPMI-1640 medium (Gibco, Life Technologies, Waltham, MA, USA) including L-glutamine, supplemented with $10 \%(\mathrm{v} / \mathrm{v})$ fetal calf serum (Gibco, Life Technologies) and 1\% (v/v) penicillin/ streptomycin (100,000 U/1 penicillin, $10 \mathrm{mg} / 1$ streptomycin, Sigma-Aldrich, St. Louis, MO, USA) at $37^{\circ} \mathrm{C}$ in a humidified atmosphere containing $5 \%$ carbon dioxide.

miRNA inhibitors and miRNA mimics. Negative controls, miR-141 inhibitors and miR-141 mimics were purchased from Shanghai GenePharma Co. Ltd. (Shanghai, China). Plasmid was transfected into cells using Lipofectamine 2000 (Invitrogen) according to the manufacturer's protocol, respectively. Transfected cells were cultured for $24 \mathrm{~h}$ before analysis.

Cell viability assay. Transfected cells were seeded into 96-well plates $\left(2 \times 10^{3}\right.$ cells/well) and cell viability was assessed using the $50 \mu 1$ of 3-(4,5-dimethylthiazol-2-yl)-2,5-diphenyltetrazolium
Table I. The designed PCR primers of miR-141 and EIF4E.

\begin{tabular}{ll}
\hline Gene & \multicolumn{1}{c}{ PCR primers } \\
\hline miR-141 & F: 5'-CACATCCACCTCCTCCACATC-3' \\
& R:5'-AATGCGGCCGCAACTCAATCAACATCACCAT-3' \\
U6 & F: 5'-CTCGCTTCGGCAGCACATATACT-3' \\
& R: 5'-ACGCTTCACGAATTTGCGTGTC-3' \\
EIF4E & F: 5'-ATGGCGACTGTCGAACCG-3' \\
& R: 5'-ATTAGATTCCGTTTTCTCCTCTTCTG-3' \\
GAPDH & F: 5'-AAGGGAAGGTTGCTGGATAGG-3' \\
& R: 5'-CACATCCACCTCCTCCACATC-3' \\
\hline
\end{tabular}

Forward, F; reverse, R.

bromide (MTT) assay. After 4-h culture, DMSO was dissolved for $10 \mathrm{~min}$ and the absorbance of each well at $490 \mathrm{~nm}$ (A490) was read on a spectrophotometer.

Apoptosis analyses. Transfected cells were seeded into 6-well

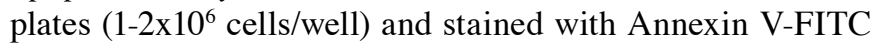
Apoptosis Detection kit (BD Biosciences, La Jolla, CA, USA) for $30 \mathrm{~min}$ in the dark. Then, cells were stained with propidium iodide (PI) solution (BD Biosciences) for $10 \mathrm{~min}$ in the dark. Cell apoptosis was measured using a FACS sorter and the data were analyzed using ModFit (both from BD Biosciences).

Caspase-3 activity. Transfected cells were seeded into 6-well plates $\left(1-2 \times 10^{6}\right.$ cells/well), extracted by cell lysis (Beyotime Institute of Biotechnology) and then centrifuged at 13,000 x g for $30 \mathrm{~min}$. Protein content was measured using BCA assay (Beyotime Institute of Biotechnology). Total cellular proteins were cultured with Ac-DEVD-pNA for $2 \mathrm{~h}$ at room temperature. The absorbance of each well at $405 \mathrm{~nm}$ was read on a spectrophotometer.

Western blotting. Transfected cells were seeded into 6-well plates $\left(1-2 \times 10^{6}\right.$ cells/well), extracted by cell lysis (Beyotime Institute of Biotechnology) and then centrifuged at 13,000 x g for $30 \mathrm{~min}$. Protein content was measured using BCA assay (Beyotime Institute of Biotechnology). Total cellular proteins were separated on an 8-10\% SDS-PAGE, transferred onto a PVDF membrane (BD Biosciences). Membrane was incubated with primary antibodies against EIF4E (1:500, Cell Signaling Technology), VEGF (1:500, Cell Signaling Technology), c-Myc (1:500, Cell Signaling Technology), Bax (1:500, Cell Signaling Technology) and GAPDH (1:500, Cell Signaling Technology) at 1:500 overnight at $4^{\circ} \mathrm{C}$. Membrane was washed with TBSA containing 5\% skim milk powder for $1 \mathrm{~h}$ at room temperature and then with anti-rabbit $\operatorname{IgG}(1: 5000$, Cell Signaling Technology) for $1 \mathrm{~h}$ at room temperature. Detection was carried out using ECL Plus Western Blotting Detection System (Amersham, UK).

Statistical analysis. All statistical analyses were performed using SPSS 19.0 software (SPSS, Inc., Chicago, IL, USA). The results are presented as mean \pm standard error. The difference between means was analyzed with ANOVA and then Student's 

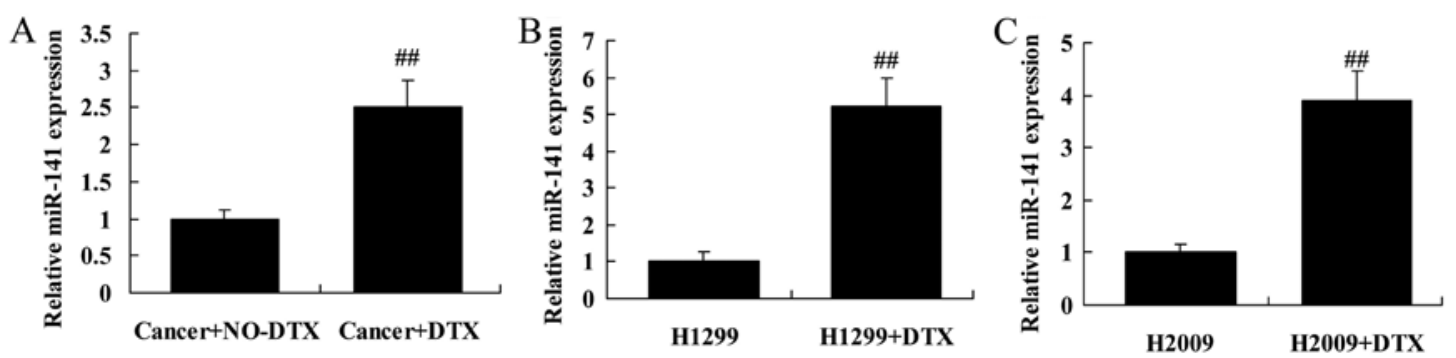

Figure 1. miR-141 expression in docetaxel chemoresistance of human NSCLC patients. The miR-141 expression in docetaxel chemoresistance of human NSCLC patients (A), H1299 cells treated by docetaxel (B) and H2009 cells treated by docetaxel (C). Cancer+NO-DTX, non-docetaxel chemoresistant patients with NSCLCs; Cancer+DTX, docetaxel chemoresistant patients with NSCLCs; H1299 cell+DTX, H1299 cells treated by docetaxel; H2009 cell+DTX, H2009 cells treated by docetaxel. ${ }^{\# \#} \mathrm{p}<0.01$ versus Cancer+NO-DTX group or ${ }^{\# \#} \mathrm{p}<0.01$ versus H1299 cell group or ${ }^{\# \#} \mathrm{p}<0.01$ versus H2009 cell group.
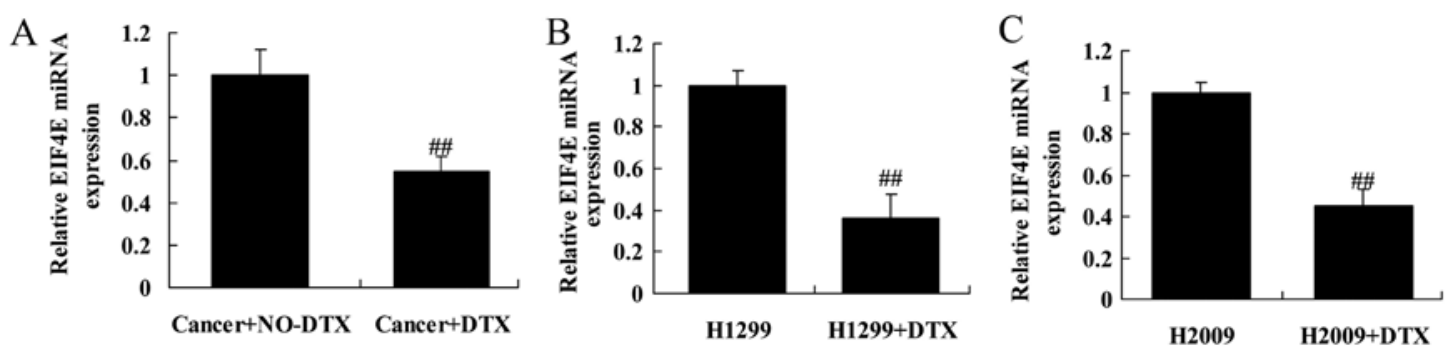

Figure 2. EIF4E expression in docetaxel chemoresistance of human NSCLC patients. The expression of EIF4E in docetaxel chemoresistance of human NSCLC patients (A), H1299 cells treated by docetaxel (B) and H1299 cell treated by docetaxel (C). Cancer+NO-DTX, non-docetaxel chemoresistant patients with NSCLCs; Cancer+ DTX, docetaxel chemoresistant patients with NSCLCs; H1299 cell+DTX, H1299 cells treated by docetaxel; H2009 cell+DTX, H2009 cells treated by docetaxel. ${ }^{\# \#} \mathrm{p}<0.01$ versus Cancer+NO-DTX group or ${ }^{\# \#} \mathrm{p}<0.01$ versus H1299 cell group or ${ }^{\# \#} \mathrm{p}<0.01$ versus H2009 cell group.
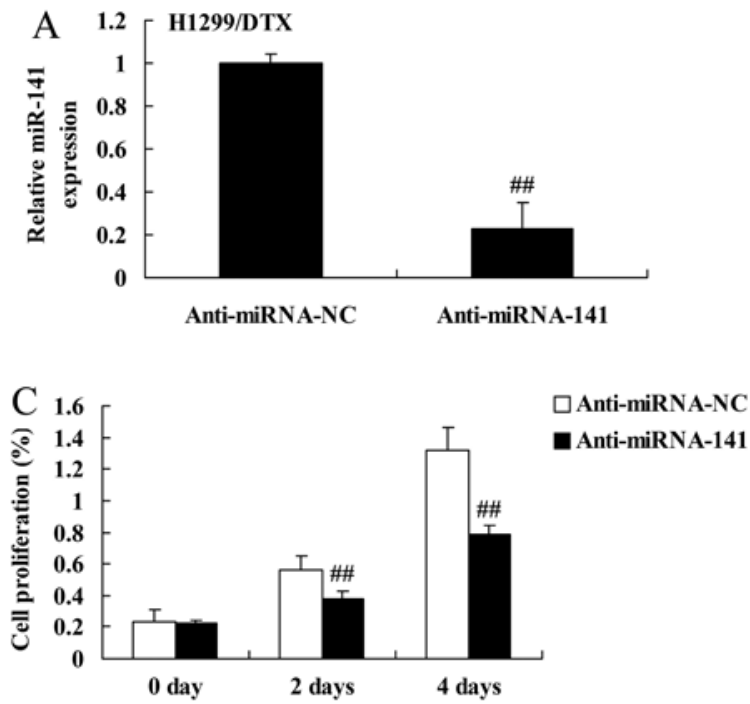
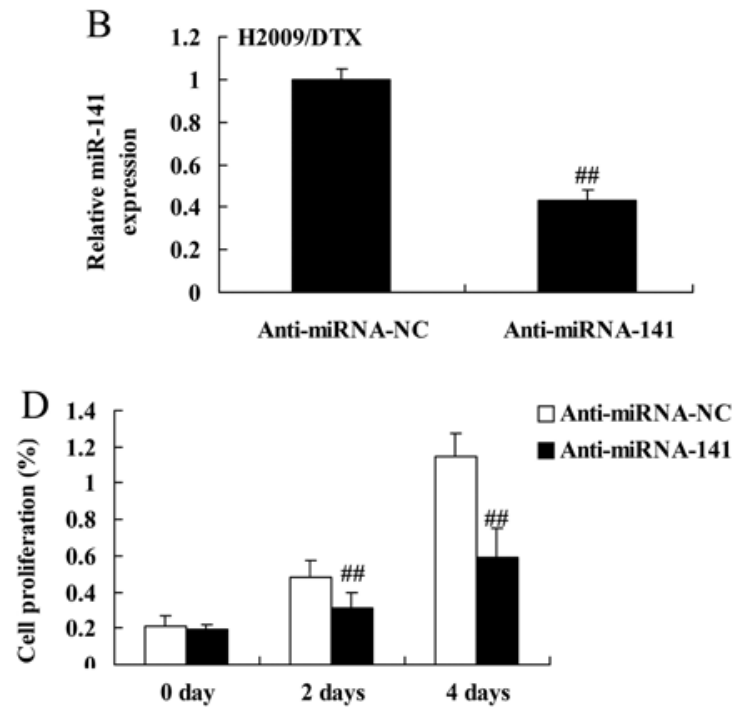

Figure 3. Downregulation of miR-141 reverses docetaxel chemoresistance of human NSCLC growth. Downregulation of miR-141 cells inhibited miR-141 expression (A and B) and cell viability (C and D) of H1299 and H2009 cells treated by docetaxel. H1299 cell+DTX, H1299 cells treated by docetaxel; H2009 cell+DTX, H2009 cell treated by docetaxel. Anti-miRNA-NC, negative controls group; Anti-miRNA-141, miR-141 inhibitors. ${ }^{\# \#}$ p $<0.01$ versus AntimiRNA-NC group.

t-test. A p-value of $<0.05$ was considered to indicate a statistically significant difference.

\section{Results}

miR-141 expression in docetaxel chemoresistance of human NSCLC patients. As shown in Fig. 1A, the miRNA expression of miR-141 in docetaxel chemoresistant patients with NSCLCs was markedly higher than those of non-docetaxel chemoresistant patients with NSCLCs. The miRNA expression of miR-141 in H1299 and H2009 cells treated by docetaxel was also higher than that of H1299 and H2009 cells treated by nondocetaxel group (Fig. 1B and C).

EIF4E expression in docetaxel chemoresistance of human NSCLC patients. Then, we found that the miRNA expression 

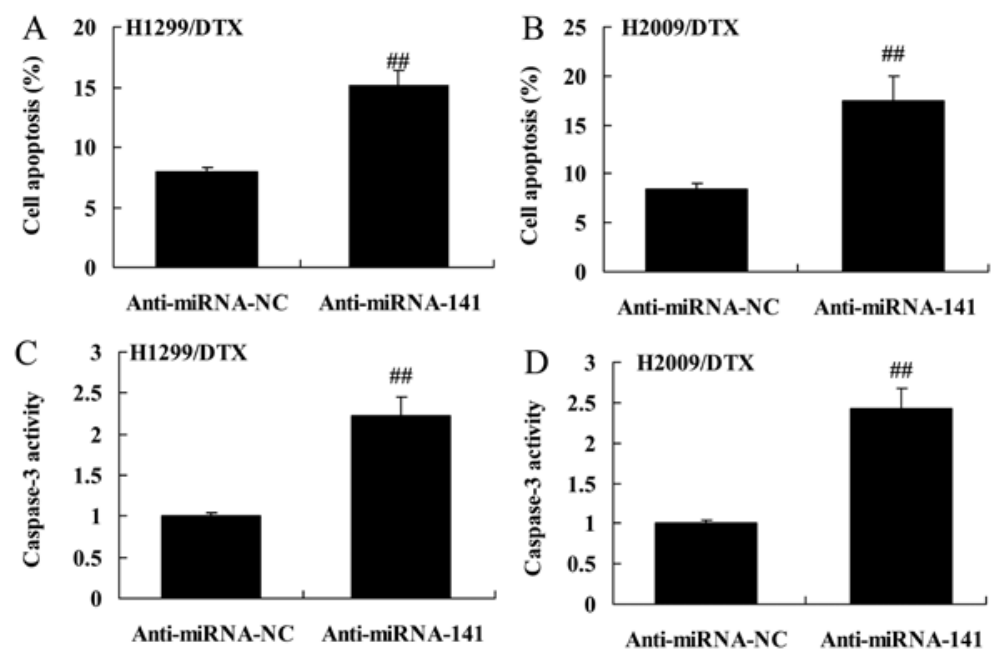

Figure 4. Downregulation of miR-141 reverses docetaxel chemoresistance of human NSCLC death and caspase-3 activity. Downregulation of miR-141 reverses docetaxel chemoresistance of human NSCLC apoptosis (A and B) and caspase-3 activity (C and D). H1299 cell+DTX, H1299 cell treated by docetaxel; H2009 cell+DTX, H2009 cells treated by docetaxel. Anti-miRNA-NC, negative controls group; Anti-miRNA-141, miR-141 inhibitors. ${ }^{\# \#<0.01 ~ v e r s u s ~ A n t i-~}$ miRNA-NC group.

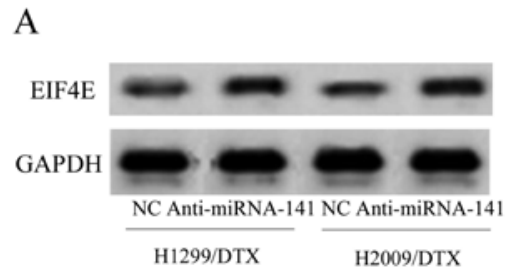

H1299/DTX H2009/DTX
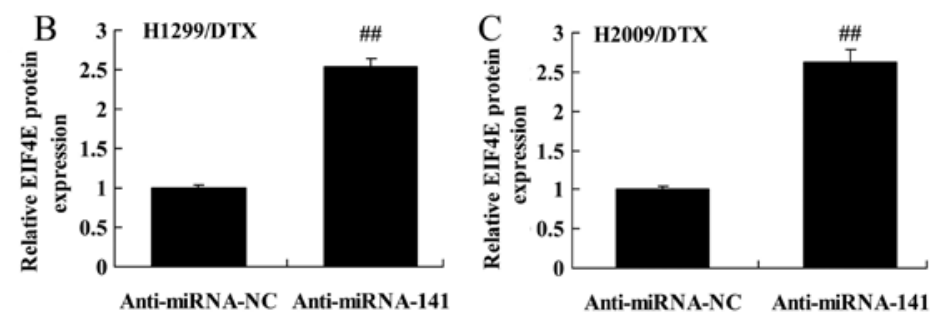

Figure 5. Downregulation of miR-141 reverses EIF4E protein expression in docetaxel chemoresistance of human NSCLC. Downregulation of miR-141 reverses EIF4E protein expression (A) and EIF4E protein expression (B and C) of statistical analysis in docetaxel chemoresistance of human NSCLC. H1299 cell+DTX, H1299 cells treated by docetaxel; H2009 cell+DTX, H2009 cells treated by docetaxel. Anti-miRNA-NC, negative controls group; Anti-miRNA-141, miR-141 inhibitors. ${ }^{\# \#} \mathrm{p}<0.01$ versus Anti-miRNA-NC group.

of EIF4E in docetaxel chemoresistant patients with NSCLCs was markedly lower than those of non-docetaxel chemoresistant patients with NSCLCs (Fig. 2A). Moreover, the miRNA expression of EIF4E in H1299 and H2009 cells treated by docetaxel was also lower than that of $\mathrm{H} 1299$ and $\mathrm{H} 2009$ cells treated by non-docetaxel group (Fig. 2B and C).

Downregulation of miR-141 reverses docetaxel chemoresistance of human NSCLC growth. Therefore, negative control and miR-141 inhibitor plasmids were transfected into H1299 and H2009 cells. MiR-141 inhibitor plasmids significantly inhibited the miR-141 expression in H1299 and H2009 cells treated by docetaxel, compared with negative control group (Fig. 3A and B). Downregulation of miR-141 significantly inhibited cell viability of H1299 and H2009 cells treated by docetaxel, compared with negative control group (Fig. 3C and D).

Downregulation of $m i R-141$ reverses docetaxel chemoresistance of human NSCLC death and caspase-3 activity. Then, we detected the downregulation of miR-141 affect on docetaxel chemoresistance of human NSCLC death and caspase-3 activity. As shown in Fig. 4A-D, the downregulation of miR-141 significantly increased cell apoptosis and caspase-3 activity of H1299 and H2009 cells treated by docetaxel, compared with negative control group.
Downregulation of $m i R-141$ reverses EIF4E protein expression in docetaxel chemoresistance of human NSCLC. We evaluated whether the downregulation of miR-141 reverses EIF4E protein expression in docetaxel chemoresistance of human NSCLC. Downregulation of miR-141 significantly induced the protein expression of EIF4E in H1299 and H2009 cells treated by docetaxel, compared with negative control group (Fig. 5A-C).

Downregulation of $\mathrm{miR}-141$ reverses VEGF protein expression in docetaxel chemoresistance of human NSCLC. To assess the mechanism of anticancer effect of miR-141 on docetaxel chemoresistance of human NSCLC, VEGF protein expression was measured using western blotting. The results from Western blot showed that the downregulation of miR-141 significantly activated the protein expression of VEGF in H1299 and H2009 cells treated by docetaxel, compared with negative control group (Fig. 6A-C).

Downregulation of $m i R-141$ reverses $c$-Myc protein expression in docetaxel chemoresistance of human NSCLC. We further assessed the mechanism of anticancer effect of miR-141 on docetaxel chemoresistance of human NSCLC, c-Myc protein expression was also measured using western blotting. Western blot showed that the downregulation of miR-141 significantly 
A

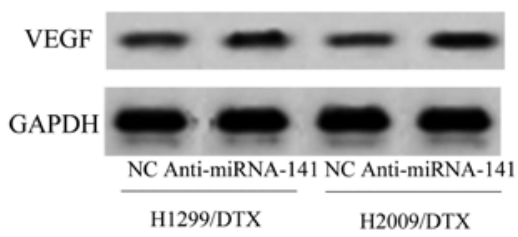

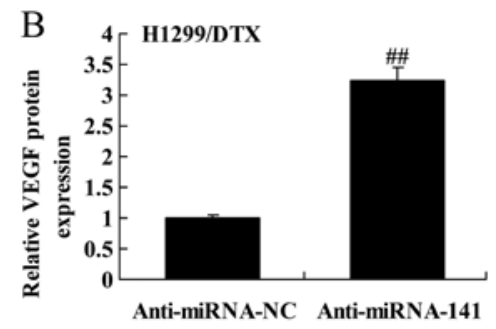

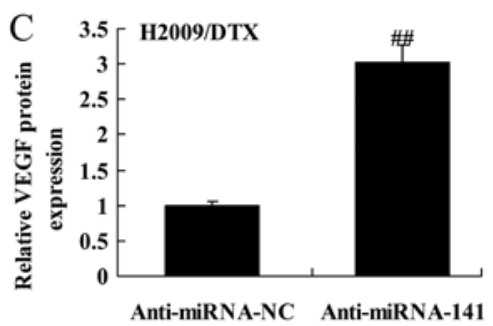

Figure 6. Downregulation of miR-141 reverses VEGF protein expression in docetaxel chemoresistance of human NSCLC. Downregulation of miR-141 reverses VEGF protein expression (A) and VEGF protein expression (B and C) of statistical analysis in docetaxel chemoresistance of human NSCLC. H1299 cell+DTX, H1299 cells treated by docetaxel; H2009 cell+DTX, H2009 cells treated by docetaxel. Anti-miRNA-NC, negative controls group; Anti-miRNA-141, miR-141 inhibitors. ${ }^{\#} \mathrm{p}<0.01$ versus Anti-miRNA-NC group.

A

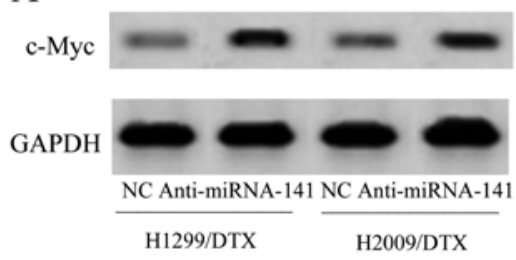

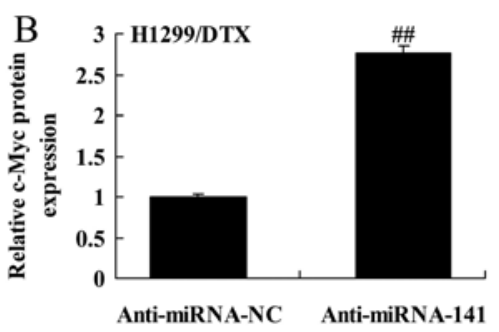

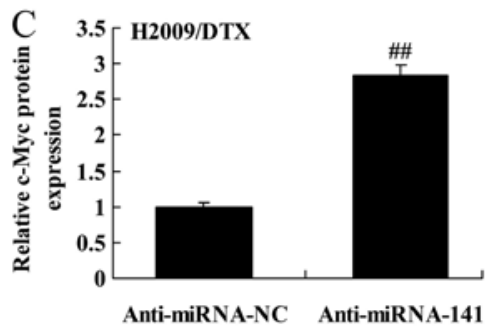

Figure 7. Downregulation of miR-141 reverses c-Myc protein expression in docetaxel chemoresistance of human NSCLC. Downregulation of miR-141 reverses c-Myc protein expression (A) and c-Myc protein expression (B and C) of statistical analysis in docetaxel chemoresistance of human NSCLC. H1299 cell+DTX, H1299 cells treated by docetaxel; H2009 cell+DTX, H2009 cells treated by docetaxel. Anti-miRNA-NC, negative controls group; Anti-miRNA-141, miR-141 inhibitors. ${ }^{\# \#} \mathrm{p}<0.01$ versus Anti-miRNA-NC group.

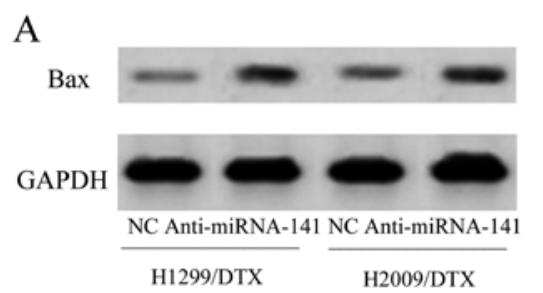

H1299/DTX

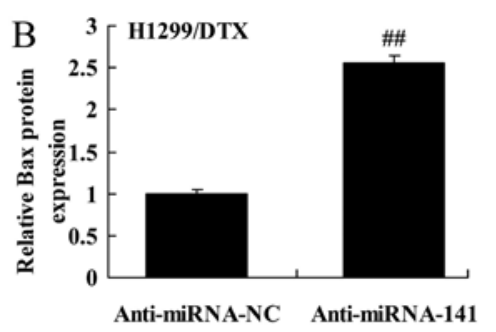

Anti-miRNA-NC Anti-miRNA-141

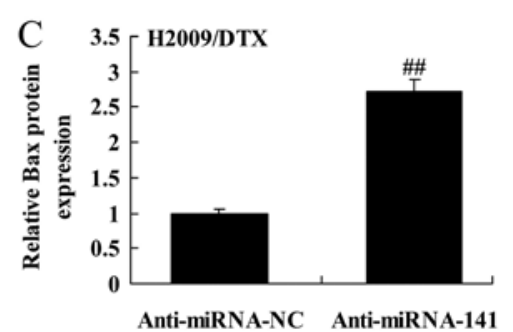

Figure 8. Downregulation of miR-141 reverses Bax protein expression in docetaxel chemoresistance of human NSCLC. Downregulation of miR-141 reverses Bax protein expression (A) and Bax protein expression (B and C) of statistical analysis in docetaxel chemoresistance of human NSCLC. H1299 cell+DTX, H1299 cells treated by docetaxel; H2009 cell+DTX, H2009 cells treated by docetaxel. Anti-miRNA-NC, negative controls group; Anti-miRNA-141, miR-141 inhibitors. ${ }^{\# \#} \mathrm{p}<0.01$ versus Anti-miRNA-NC group.

promoted the protein expression of c-Myc in H1299 and H2009 cells treated by docetaxel, compared with negative control group (Fig. 7A-C).

Downregulation of $m i R-141$ reverses Bax protein expression in docetaxel chemoresistance of human NSCLC. We explored the downregulation of miR-141 affect on Bax protein expression in docetaxel chemoresistance of human NSCLC. As shown in Fig. 8A-C, downregulation of miR-141 significantly activated Bax protein expression in H1299 and H2009 cells treated by docetaxel, compared with negative control group.

Upregulation of miR-141 reverses docetaxel chemoresistance of human NSCLC growth. On the contrary, we used miR-141 mimics to increase miR-141 expression in H1299 and H2009 cells treated by docetaxel, which was used to analyze the upregulation of miR-141 affect on docetaxel chemoresistance of human NSCLC growth. As shown in Fig. 9A and B, miR-141 mimics significantly increased the miR-141 expression in H1299 and H2009 cells treated by docetaxel. In addition, the upregulation of miR-141 significantly increased cell viability of H1299 and H2009 cells treated by docetaxel, compared with negative control group (Fig. 9C and D).

Upregulation of miR-141 reverses docetaxel chemoresistance of human NSCLC death and caspase-3 activity. We confirmed whether the upregulation of miR-141 reverses docetaxel chemoresistance of human NSCLC death and caspase-3 activity. Upregulation of miR-141 significantly inhibited cell apoptosis and caspase-3 activity of H1299 and H2009 cells treated by docetaxel, compared with negative control group (Fig. 10A-D).

Upregulation of miR-141 reverses EIF4E protein expression in docetaxel chemoresistance of human NSCLC. To further 

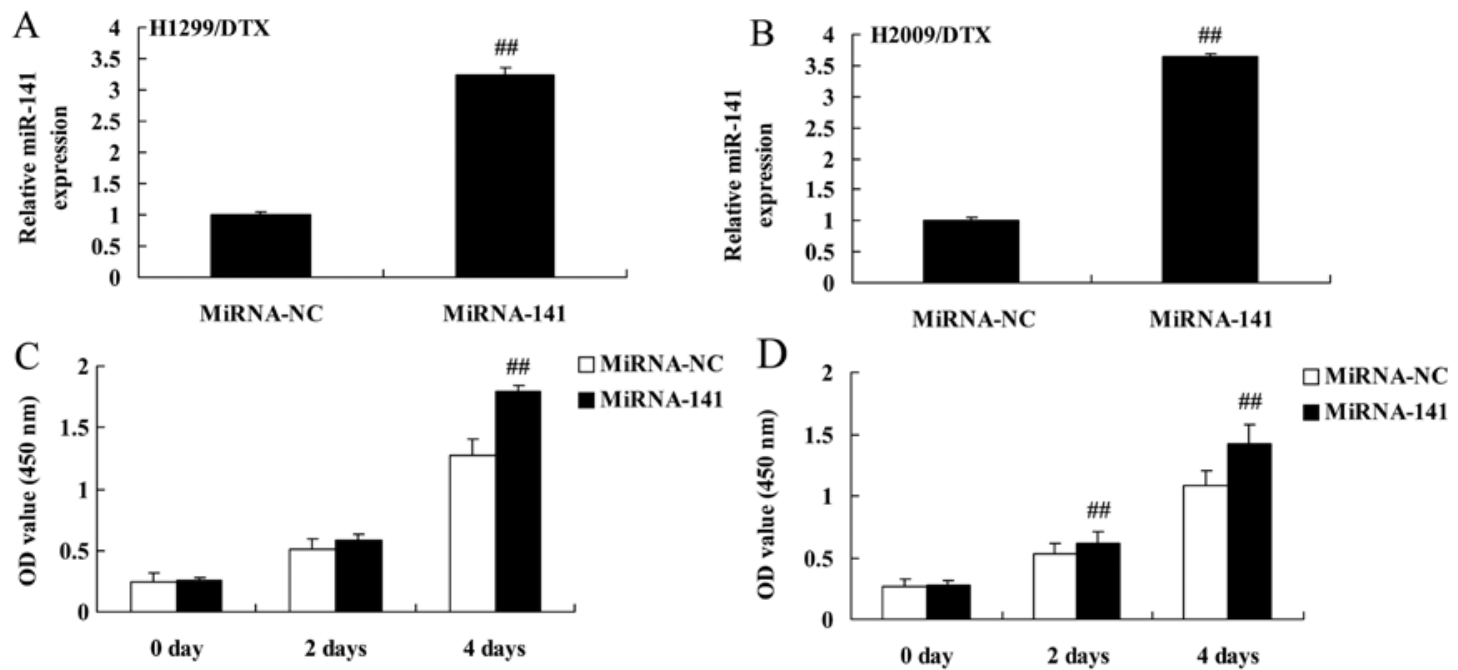

Figure 9. Upregulation of miR-141 reverses docetaxel chemoresistance of human NSCLC growth. Upregulation of miR-141 cell reverses miR-141 expression (A and B) and cell viability (C and D) of H1299 and H2009 cells treated by docetaxel. H1299 cell+DTX, H1299 cells treated by docetaxel; H2009 cell+DTX, H2009 cells treated by docetaxel. miRNA-NC, negative controls group; miRNA-141, miR-141 mimics. ${ }^{\# \#}$ p $<0.01$ versus Anti-miRNA-NC group.
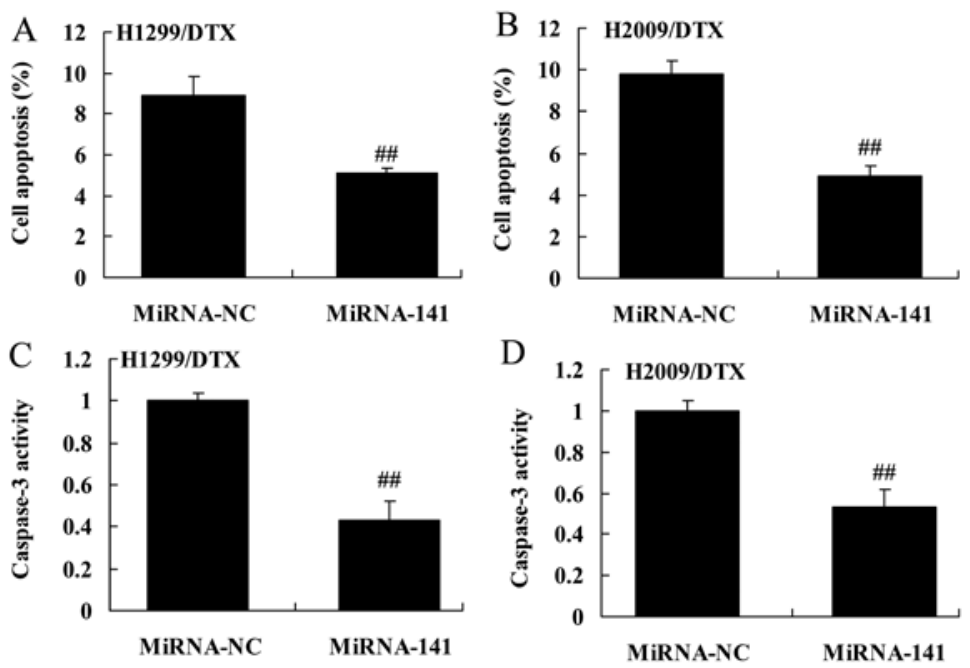

Figure 10. Upregulation of miR-141 reverses docetaxel chemoresistance of human NSCLC death and caspase-3 activity. Upregulation of miR-141 reverses docetaxel chemoresistance of human NSCLC apoptosis (A and B) and caspase-3 activity (C and D). H1299 cell+DTX, H1299 cells treated by docetaxel; H2009

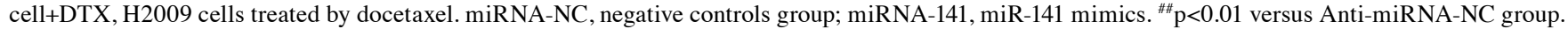
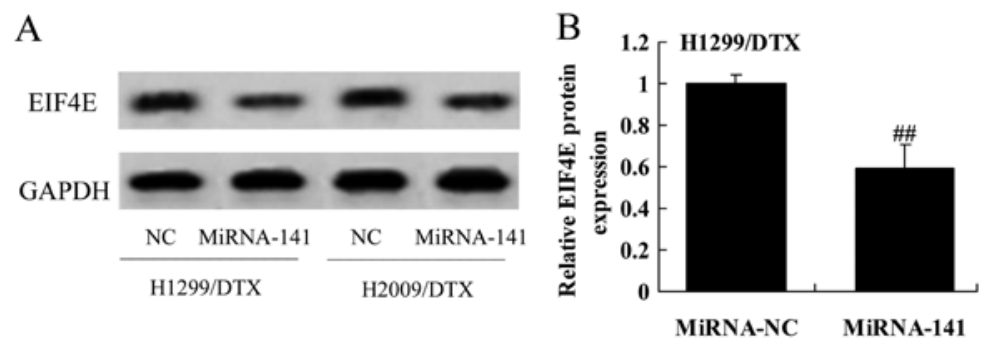

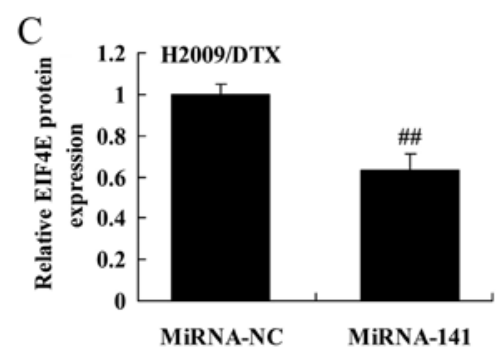

Figure 11. Upregulation of miR-141 reverses EIF4E protein expression in docetaxel chemoresistance of human NSCLC. Upregulation of miR-141 reverses EIF4E protein expression (A) and EIF4E protein expression (B and C) of statistical analysis in docetaxel chemoresistance of human NSCLC. H1299 cell+DTX, H1299 cells treated by docetaxel; H2009 cell+DTX, H2009 cells treated by docetaxel. miRNA-NC, negative controls group; miRNA-141, miR-141 mimics.

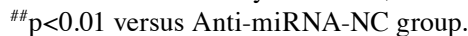

confirm that the upregulation of miR-141 reverses EIF4E protein expression in docetaxel chemoresistance of human NSCLC. In contrast, the EIF4E protein expression of H1299 and $\mathrm{H} 2009$ cells treated by docetaxel was significantly inhibited by upregulation of miR-141, compared with negative control group (Fig. 11A-C). 

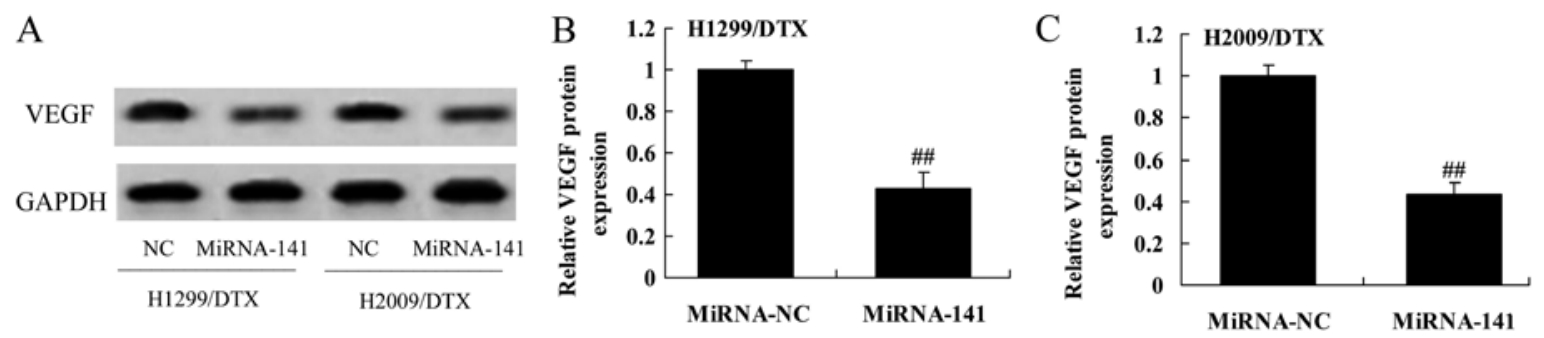

Figure 12. Upregulation of miR-141 reverses VEGF protein expression in docetaxel chemoresistance of human NSCLC. Upregulation of miR-141 reverses EIF4E protein expression (A) and EIF4E protein expression (B and C) of statistical analysis in docetaxel chemoresistance of human NSCLC. H1299 cell+DTX, H1299 cells treated by docetaxel; H2009 cell+DTX, H2009 cells treated by docetaxel. miRNA-NC, negative controls group; miRNA-141, miR-141 mimics. ${ }^{\#} \mathrm{p}<0.01$ versus Anti-miRNA-NC group.

A

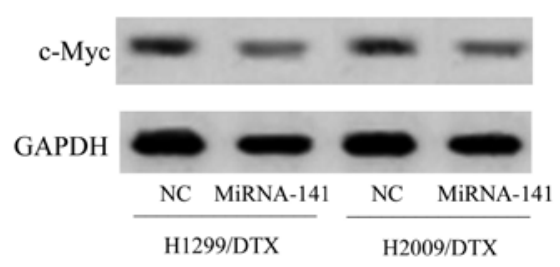

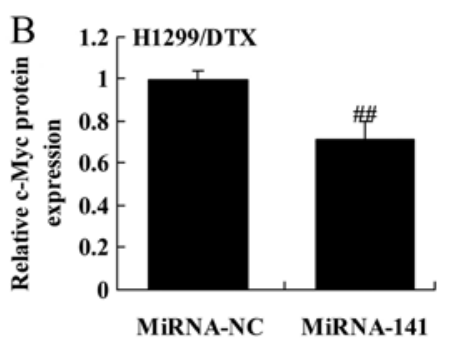

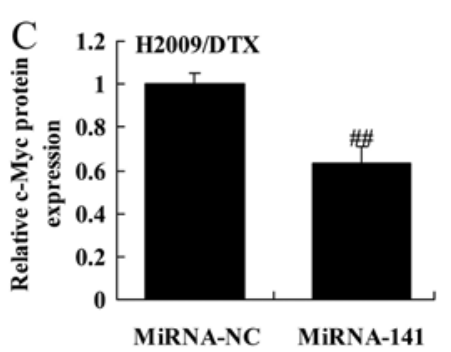

Figure 13. Upregulation of miR-141 reverses c-Myc protein expression in docetaxel chemoresistance of human NSCLC. Upregulation of miR-141 reverses c-Myc protein expression (A) and EIF4E protein expression (B and C) of statistical analysis in docetaxel chemoresistance of human NSCLC. H1299 cell+DTX, H1299 cells treated by docetaxel; H2009 cell+DTX, H2009 cells treated by docetaxel. miRNA-NC, negative controls group; miRNA-141, miR-141 mimics. ${ }^{\# \#} \mathrm{p}<0.01$ versus Anti-miRNA-NC group.

A

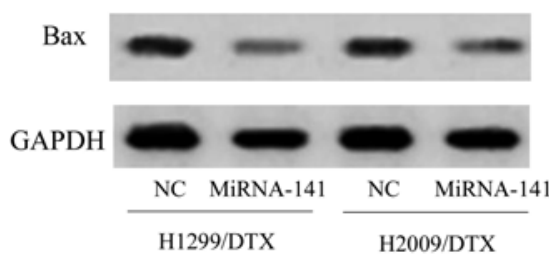

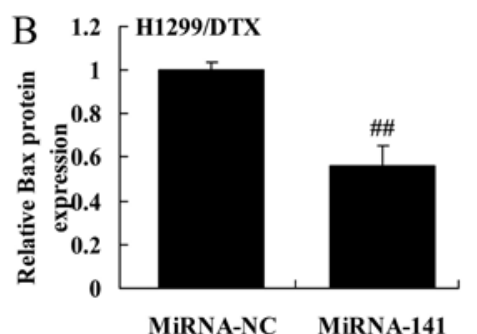

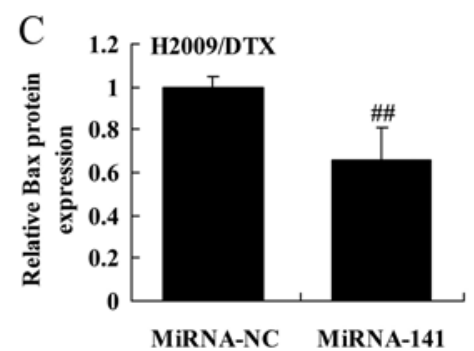

Figure 14. Upregulation of miR-141 reverses Bax protein expression in docetaxel chemoresistance of human NSCLC. Upregulation of miR-141 reverses Bax protein expression (A) and Bax protein expression (B and C) of statistical analysis in docetaxel chemoresistance of human NSCLC. H1299 cell+DTX, H1299 cells treated by docetaxel; H2009 cell+DTX, H2009 cells treated by docetaxel. miRNA-NC, negative controls group; miRNA-141, miR-141 mimics. ${ }^{\# \#}$ p $<0.01$ versus Anti-miRNA-NC group.

Upregulation of miR-141 reverses VEGF protein expression in docetaxel chemoresistance of human NSCLC. To measure VEGF protein expression in docetaxel chemoresistance of human NSCLC following upregulation of miR-141, VEGF protein was measured using western blotting. Upregulation of miR-141 significantly suppressed VEGF protein of H1299 and $\mathrm{H} 2009$ cells treated by docetaxel, compared with negative control group (Fig. 12A-C).

Upregulation of miR-141 reverses c-Myc protein expression in docetaxel chemoresistance of human NSCLC. We measured c-Myc protein expression in docetaxel chemoresistance of human NSCLC using western blotting after upregulation of miR-141. The results showed a significant inhibition in c-Myc protein expression of $\mathrm{H} 1299$ and $\mathrm{H} 2009$ cells treated by docetaxel after upregulation of miR-141 than the control cells (Fig. 13A-C).
Upregulation of miR-141 reverses Bax protein expression in docetaxel chemoresistance of human NSCLC. Next, we measured whether the upregulation of miR-141 reverses Bax protein expression in docetaxel chemoresistance of human NSCLC. As expected, Bax protein expression of H1299 and H2009 cells treated by docetaxel significantly suppressed the protein expression of Bax, compared with negative control group (Fig. 14A-C).

\section{Discussion}

Lung cancer is one of the most common malignant tumors. It is a malignant tumor with the highest fatality rate as well. According to histologic classification, $80 \%$ of lung cancer is non-small cell lung cancer (NSCLC) (2). Due to the absence of effective early diagnosis method, most of the patients at preliminary diagnosis have lost the opportunity 
for surgery (13). In consequence, chemotherapy has become the main treatment method of NSCLC (14). Docetaxel, as a third generation cytotoxic drug, has been applied on first-line therapy for NSCLC at advanced stage (15). Similar to other chemotherapeutics, the increasing drug resistance also affects its clinical application and curative effect of chemotherapy (4). Therefore, to illuminate its resistance mechanism has important realistic significance on selection of therapeutic strategy, preventing or reversing the drug resistance to the maximum extent and improving curative effect of chemotherapy. Our work demonstrates that miR-141 expression in docetaxel chemoresistant patients with NSCLCs was markedly higher than those of non-docetaxel chemoresistant patients with NSCLCs. Downregulation of miR-141 significantly inhibited cell viability, and increased cell apoptosis and caspase-3 activity of H1299 and H2009 cells treated by docetaxel.

Experiments have shown that many malignant tumors in clinic are accompanied by overexpression of eIF4E (5). In addition, its expression level is closely related to differentiation degree of malignant tumors and metastasis. In the past 10 years, the cap binding protein eIF4E of mRNA has obtained increased attention during tumorigenesis and the development process (16). High expression of eIF4E exists on a variety of malignant tumors, such as breast cancer, laryngeal carcinoma, esophagus cancer, thyroid cancer, gastric cancer, lung cancer and leukemia. Furthermore, its expression degree is related to tumor malignant degree (17). High expression of eIF4E can obviously affect normal cell phenotype, induce cell proliferation activity, promote cell conversion and lead to genesis and metastasis of tumor (16). Transfer of eIF4E antisense RNA to the cell or inhibition of its combination with competitiveness of eIF4E binding proteins, will reduce protein translation, inhibit cell transformation, reduce tumorigenesis, alleviate infiltration and metastasis (18). In the present study, we showed that the expression of EIF4E in docetaxel chemoresistant patients with NSCLCs was markedly lower than those of non-docetaxel chemoresistant patients with NSCLCs. Downregulation of miR-141 significantly induced the protein expression of EIF4E in H1299 and H2009 cells treated by docetaxel. Yao et al reported that miR-141 regulates EIF4E expression in docetaxel chemoresistance of breast cancer cells (19). These data suggest that miR-141 is a potential target for chemosensitizing BCs.

It has been detected that overexpression of $\mathrm{dF} 4 \mathrm{E}$ exists in a variety of malignant tumors (8). In addition, the overexpression is positively correlated to infiltration and metastasis ability of tumor. Overexpression of eIF4E can selectively strengthen the expression of a series of malignant proteins. Such malignant proteins include angiogenesis protein (such as FGF and VEGF), proliferation adherence and infiltrating related proteins. VEGF is a upregulated protein after overexpression of eIF4E (18). It is a key effective factor during angiogenesis process. Angiogenesis will increase after overexpression of VEGF which can provide adequate oxygen and nutrients for ectopic endometrium and promote adhesion and plant growth of ectopic cells; new vessels can produce and secrete a variety of cytokines, growth factors and degradation enzymes thus accelerate adherence infiltration of ectopic endometrium; amplification of new blood vessels in endothelial cells provides opportunity for distant metastasis of ectopic endometrium via circulatory system $(20,21)$. Importantly, our present results showed that downregulation of miR-141 significantly activated the protein expression of VEGF in H1299 and H2009 cells treated by docetaxel. Tejero et al showed that miR-141 regulates VEGF in early stage non-small cell lung cancer adenocarcinoma (22).

It has been proven by overexpression of eIF4E in breast cancer, that NSCLC and prostatic cancer instead of typical benign lesions overexpression of eIF4E may be an important mark at the malignant transformation process (23). A large number of proteins shall be generated to maintain rapid proliferation of tumor cells. It has been detected in the cells from eIF4E that synthesis of only few proteins has been significantly improved (24). The synthesis of most proteins slightly increased. In vitro experiment has verified that the 'minority proteins' of cells with overexpression of eIF4E include: CCND1, c-myc and ODC, which can regulate cell cycle process and promote tumorigenesis, as well as promoters for fibroblast growth factor and vascular endothelial cell growth factor (25). Our work also demonstrates that downregulation of miR-141 significantly promoted the protein expression of c-Myc in H1299 and H2009 cells treated by docetaxel. Zhang et al proposed that microRNA-141 is involved in nasopharyngeal carcinomarelated gene through c-Myc pathways (26).

Bax is the first member of bcl-2 being confirmed with cell apoptosis promotion effect (27). In case of high expression of Bax protein, it can form Bax homodimer and/or Bax-Bcl-2 heterodimer with Bcl-2 thus inhibiting Bcl-2 functions, accelerating apoptosis (28). The expression of Bcl-2 and Bax, as well as the proportion of the two (Bax/Bcl-2) have key effects on the survival of cells after accepting apoptosis signal (29). In case of overexpression of Bcl-2, the cell will survive; and in case of overexpression of Bax, the reaction of cell on death signal will be enhanced and cell death will increase (23). In this study, we found that downregulation of miR-141 significantly activated Bax protein expression in H1299 and H2009 cells treated by docetaxel. Upregulation of miR-141 suppressed EIF4E, VEGF, c-Myc protein expression and inhibited Bax in H1299 or H2009/docetaxel cells.

In conclusion, the results of the present study demonstrated that miR-141 suppressed cell proliferation, induced cell death and increased caspase-3 activity in H1299 or H2009/docetaxel cell via EIF4E, VEGF, c-Myc and Bax signaling pathway, and suggests that miR-141 may serve as a biomarker for response to chemotherapy in NSCLCs.

\section{References}

1. Louie AV, van Werkhoven E, Chen H, Smit EF, Paul MA, Widder J, Groen HJ, van den Borne BE, De Jaeger K, Slotman BJ, et al: Patient reported outcomes following stereotactic ablative radiotherapy or surgery for stage IA non-small-cell lung cancer: Results from the ROSEL multicenter randomized trial. Radiother Oncol 117: 44-48, 2015.

2. Aisner J, Manola JB, Dakhil SR, Stella PJ, Sovak MA and Schiller JH: Vandetanib plus chemotherapy for induction followed by vandetanib or placebo as maintenance for patients with advanced non-small-cell lung cancer: A randomized phase 2 PrECOG study (PrE0501). J Thorac Oncol 8: 1075-1083, 2013.

3. Lal R, Bourayou N, Hillerdal G, Nicolson M, Vikstrom A, Lorenzo M, D'yachkova Y, Barriga S and Visseren-Grul C: Home administration of maintenance pemetrexed for patients with advanced non-squamous non-small cell lung cancer: Rationale, practicalities and phase II feasibility study design. Health Qual Life Outcomes 11: 163, 2013. 
4. Zhu Z, Liu W, Gillin M, Gomez DR, Komaki R, Cox JD, Mohan R and Chang JY: Assessing the robustness of passive scattering proton therapy with regard to local recurrence in stage III non-small cell lung cancer: A secondary analysis of a phase II trial. Radiat Oncol 9: 108, 2014.

5. Graff JR, Boghaert ER, De Benedetti A, Tudor DL, Zimmer CC, Chan SK and Zimmer SG: Reduction of translation initiation factor 4E decreases the malignancy of ras-transformed cloned rat embryo fibroblasts. Int J Cancer 60: 255-263, 1995.

6. Rosenwald IB, Hutzler MJ, Wang S, Savas L and Fraire AE: Expression of eukaryotic translation initiation factors $4 \mathrm{E}$ and 2alpha is increased frequently in bronchioloalveolar but not in squamous cell carcinomas of the lung. Cancer 92: 2164-2171, 2001.

7. Kaufman JL, Glockner F, Chang BB, Koslow AR, Shah DM and Leather RP: Impact of the presence of orthopedic hardware on technical performance of major amputations. Ann Vasc Surg 4: 356-358, 1990.

8. Chang SH, Kim JE, Lee JH, Minai-Tehrani A, Han K, Chae C, Cho YH, Yun JH, Park K, Kim YS, et al: Aerosol delivery of eukaryotic translation initiation factor 4E-binding protein 1 effectively suppresses lung tumorigenesis in K-rasLA1 mice. Cancer Gene Ther 20: 331-335, 2013.

9. Zagryazhskaya A and Zhivotovsky B: miRNAs in lung cancer: A link to aging. Ageing Res Rev 17: 54-67, 2014.

10. Yamashita R, Sato M, Kakumu T, Hase T, Yogo N, Maruyama E, Sekido Y, Kondo M and Hasegawa Y: Growth inhibitory effects of miR-221 and miR-222 in non-small cell lung cancer cells. Cancer Med 4: 551-564, 2015.

11. Ye XW, Yu H, Jin YK, Jing XT, Xu M, Wan ZF and Zhang XY: miR-138 inhibits proliferation by targeting 3-phosphoinositidedependent protein kinase-1 in non-small cell lung cancer cells. Clin Respir J 9: 27-33, 2015.

12. Zhu X, Li D, Yu F, Jia C, Xie J, Ma Y, Fan S, Cai H, Luo Q, Lv Z, et al: miR-194 inhibits the proliferation, invasion, migration, and enhances the chemosensitivity of non-small cell lung cancer cells by targeting forkhead box A1 protein. Oncotarget 7: 13139-13152, 2016.

13. Komiyama K, Kobayashi K, Minezaki S, Kotajima F, Sutani A, Kasai T, Mori K, Hoshi E, Takayanagi N, Koyama S, et al; Kanto Respiratory Disease Study Group: Phase I/II trial of a biweekly combination of S-1 plus docetaxel in patients with previously treated non-small cell lung cancer (KRSG-0601). Br J Cancer 107: 1474-1480, 2012.

14. Wang L, Wu S, Ou G, Bi N, Li W, Ren H, Cao J, Liang J, Li J, Zhou Z, et al: Randomized phase II study of concurrent cisplatin/ etoposide or paclitaxel/carboplatin and thoracic radiotherapy in patients with stage III non-small cell lung cancer. Lung Cancer 77: 89-96, 2012

15. Shukuya T, Ko R, Mori K, Kato M, Yagishita S, Kanemaru R, Honma Y, Shibayama R, Koyama R, Shimada N, et al: Prognostic factors in non-small cell lung cancer patients who are recommended to receive single-agent chemotherapy (docetaxel or pemetrexed) as a second- or third-line chemotherapy: In the era of oncogenic drivers and molecular-targeted agents. Cancer Chemother Pharmacol 76: 771-776, 2015.

16. Satheesha S, Cookson VJ, Coleman LJ, Ingram N, Madhok B, Hanby AM, Suleman CA, Sabine VS, Macaskill EJ, Bartlett JM, et al: Response to mTOR inhibition: Activity of eIF4E predicts sensitivity in cell lines and acquired changes in eIF4E regulation in breast cancer. Mol Cancer 10: 19, 2011.
17. Mizutani R, Imamachi N, Suzuki Y, Yoshida H, Tochigi N, Oonishi T, Suzuki Y and Akimitsu N: Oncofetal protein IGF2BP3 facilitates the activity of proto-oncogene protein eIF4E through the destabilization of EIF4E-BP2 mRNA. Oncogene 35: 3495-3502, 2016.

18. Robichaud N, del Rincon SV, Huor B, Alain T, Petruccelli LA, Hearnden J, Goncalves C, Grotegut S, Spruck CH, Furic L, et al: Phosphorylation of eIF4E promotes EMT and metastasis via translational control of SNAIL and MMP-3. Oncogene 34: 2032-2042, 2015.

19. Yao YS, Qiu WS, Yao RY, Zhang Q, Zhuang LK, Zhou F, Sun LB and Yue L: miR-141 confers docetaxel chemoresistance of breast cancer cells via regulation of EIF4E expression. Oncol Rep 33: 2504-2512, 2015 .

20. Smith KA, Zhou B, Avdulov S, Benyumov A, Peterson M, Liu Y, Okon A, Hergert P, Braziunas J, Wagner CR, et al: Transforming growth factor- $\beta 1$ induced epithelial mesenchymal transition is blocked by a chemical antagonist of translation factor eIF4E. Sci Rep 5: 18233, 2015.

21. Inglis DJ, Lavranos TC, Beaumont DM, Leske AF, Brown CK, Hall AJ and Kremmidiotis G: The vascular disrupting agent BNC105 potentiates the efficacy of VEGF and mTOR inhibitors in renal and breast cancer. Cancer Biol Ther 15: 1552-1560, 2014

22. Tejero R, Navarro A, Campayo M, Viñolas N, Marrades RM, Cordeiro A, Ruíz-Martínez M, Santasusagna S, Molins L, Ramirez J, et al: miR-141 and miR-200c as markers of overall survival in early stage non-small cell lung cancer adenocarcinoma. PLoS One 9: e101899, 2014.

23. Li J, Huang Y, Gao Y, Wu H, Dong W and Liu L: Antibiotic drug rifabutin is effective against lung cancer cells by targeting the eIF4E- $\beta$-catenin axis. Biochem Biophys Res Commun 472: 299-305, 2016

24. Li L, Zhang L, Liu D, Cheng Y, Jing YT, Yu H, Zhou P, Song J and Li WM: Overexpression of eukaryotic translation initiation factor 4E-binding protein 1 induces the alteration of immune status in H1299 lung cancer cells. Thorac Cancer 6: 427-432, 2015.

25. Li S, Jia Y, Jacobson B, McCauley J, Kratzke R, Bitterman PB and Wagner CR: Treatment of breast and lung cancer cells with a N-7 benzyl guanosine monophosphate tryptamine phosphoramidate pronucleotide (4Ei-1) results in chemosensitization to gemcitabine and induced eIF4E proteasomal degradation. Mol Pharm 10: 523-531, 2013

26. Zhang L, Deng T, Li X, Liu H, Zhou H, Ma J, Wu M, Zhou M, Shen S, Li X, et al: microRNA-141 is involved in a nasopharyngeal carcinoma-related genes network. Carcinogenesis 31: 559-566, 2010.

27. Jiang H, Zhao PJ, Su D, Feng J and Ma SL: Paris saponin I induces apoptosis via increasing the $\mathrm{Bax} / \mathrm{Bcl}-2$ ratio and caspase- 3 expression in gefitinib-resistant non-small cell lung cancer in vitro and in vivo. Mol Med Rep 9: 2265-2272, 2014

28. Lee SH, Kim DY, Jing F, Kim H, Yun CO, Han DJ and Choi EY: Del-1 overexpression potentiates lung cancer cell proliferation and invasion. Biochem Biophys Res Commun 468: 92-98, 2015.

29. You BR, Shin HR and Park WH: PX-12 inhibits the growth of A549 lung cancer cells via G2/M phase arrest and ROS-dependent apoptosis. Int J Oncol 44: 301-308, 2014. 